\title{
JET OVER A HOT SPHERE
}

\author{
Jian-Jun SHU*
}

\section{ABSTRACT}

The paper considers heat transfer characteristics of thin film flow over a hot sphere from a cold vertical jet of liquid falling onto the surface. A numerical solution of high accuracy is obtained for large Reynolds numbers using the modified Keller box method. A good agreement is obtained.

\section{KEY WORDS}

Heat Transfer, thin film flow, large Reynolds numbers and Keller box method.

\footnotetext{
*Associate Professor, School of Mechanical \& Aerospace Engineering, Nanyang Technological University, 50 Nanyang Avenue, Singapore 639798.
} 


\section{INTRODUCTION}

The flow of a thin liquid film under gravity over a sphere occurs frequently in a variety of industrial heat-transfer applications, such as heat exchange in coating operations, in high precision wetted spheres and in multiple sphere absorbers. In order to understand the operations and, in particular, the efficiency of these processes, it is important to have a detailed study of such flows. However previous studies of thin film flow over a sphere were confined purely to the hydrodynamic problem. Gyure \& Krantz [1] used a perturbation analysis for low Reynolds numbers. Gribben [2] obtained an approximation using the Pohlhausen integral momentum technique, which assumed an approximate velocity profile across the thickness of the film; Hunt [3] obtained a numerical solution using the modified Keller box method, which accommodated the outer, free boundary. Heat-transfer characteristics of the flow have not been considered.

In this paper an accurate numerical solution for both velocity and temperature distributions is obtained. The heat transfer in the flow of a cold, axisymmetric jet over a hot sphere is investigated here.

\section{MODELLING}

The problem to be examined concerns the film cooling which occurs when a cold vertically draining column strikes a hot sphere. Although a column of fluid draining under gravity is accelerated and thin at impact, it is reasonable to model the associated volume flow as a jet of uniform velocity $U_{0}$ and radius $H_{0}$ as is illustrated in Fig. 1. The notation $Q=\pi H_{0}^{2} U_{0}$ is introduced for the flow rate and a film Reynolds number may be defined as $R_{e}=\frac{U_{0} a}{v}$ based on the sphere radius where $v$ is the kinematic viscosity of the fluid and $a$ is the radius of the sphere.

\section{GOVERNING EQUATIONS}

The flow under investigation has been modelled as a steady, axisymmetric flow of incompressible fluid. In the absence of viscous dissipation, the equations expressing conservation of mass, momentum and energy are consequently

$$
\begin{gathered}
\nabla \bullet \vec{V}=0 \\
\rho(\vec{V} \bullet \nabla) \vec{V}=\rho \vec{F}-\nabla P+\mu \nabla^{2} \vec{V} \\
\rho C_{p}(\vec{V} \bullet \nabla) T=k \nabla^{2} T
\end{gathered}
$$

where $\vec{V}=\left(v_{\theta}, v_{r}\right)$ are velocity components associated with spherical coordinates $(\theta, r)$ measured the angular displacement from the top of the sphere and the radial distance from the centre of the sphere respectively. $\rho, \mu, C_{p}$ and $k$ are the density, dynamic viscosity, specific heat at constant pressure and the thermal 
conductivity of the cooling fluid in the jet respectively. $T$ and $P$ are respectively the temperature and pressure within the fluid.

In the specified physical setting, the equations are to be solved subject to the following conditions.

(i) The no slip boundary condition at the wall requires that

$$
v_{\theta}=v_{r}=0 \text { on } r=a, 0 \leq \theta \leq \pi .
$$

(ii) The temperature at the wall is assumed constant as $T_{w}$, say

$$
\text { i.e. } T=T_{w} \text { on } r=a, 0 \leq \theta \leq \pi \text {. }
$$

(iii) On the free surface of the film, prescribed by $r=a+H(\theta)$, the shearing stress may be assumed negligible and consequently

$$
\frac{\partial v_{\theta}}{\partial r}=0 \text { at } r=a+H(\theta), 0 \leq \theta \leq \pi .
$$

(iv) Similarly, in a film cooling environment such as water surrounded by air, it may be assumed that there is negligible heat flux on the free surface and hence that

$$
\frac{\partial T}{\partial r}=0 \quad \text { at } r=a+H(\theta), 0 \leq \theta \leq \pi .
$$

(v) Once an overall flow rate $Q=\pi H_{0}^{2} U_{0}$ has been prescribed, a conservation of volume flow constraint at any given $\theta$ station leads to the condition

$$
2 \pi \sin \theta \int_{a}^{a+H(\theta)} r v_{\theta}(\theta, r) d r=\text { constant }=\pi H_{0}^{2} U_{0} \quad \text { for } \quad 0 \leq \theta \leq \pi .
$$

Under the assumption that the film thickness remains thin relative to a characteristic horizontal dimension, a boundary layer treatment of the equations leads to significant simplification.

The following non-dimensional variables are introduced

$$
\begin{array}{r}
x=\theta, \bar{Y}=\frac{R_{e}^{\frac{1}{2}}(r-a)}{a}, \bar{H}(x)=\frac{R_{e}^{\frac{1}{2}} H(\theta)}{a}, \\
\bar{U}=\frac{v_{\theta}}{U_{0}}, \bar{V}=\frac{R_{e}^{\frac{1}{2}} v_{r}}{U_{0}}, \bar{\phi}=\frac{T-T_{w}}{T_{0}-T_{w}}, p=\frac{P}{\rho U_{0}^{2}} .
\end{array}
$$

In the limit $R_{e} \rightarrow+\infty$ with $x$ remaining $O(1)$ and after neglecting terms of $o\left(\frac{1}{R_{e}}+\frac{H_{0}^{2}}{a^{2}}\right)$ compared with unity, the following equations are obtained

$$
\begin{gathered}
\frac{\partial}{\partial x}(\bar{U} \sin x)+\frac{\partial}{\partial \bar{Y}}(\bar{V} \sin x)=0 \\
\bar{U} \frac{\partial \bar{U}}{\partial x}+\bar{V} \frac{\partial \bar{U}}{\partial \bar{Y}}=\frac{1}{F_{r}} \sin x-\frac{\partial p}{\partial x}+\frac{\partial^{2} \bar{U}}{\partial \bar{Y}^{2}} \\
\frac{\partial p}{\partial \bar{Y}}=0
\end{gathered}
$$




$$
P_{r}\left(\bar{U} \frac{\partial \bar{\phi}}{\partial x}+\bar{V} \frac{\partial \bar{\phi}}{\partial \bar{Y}}\right)=\frac{\partial^{2} \bar{\phi}}{\partial \bar{Y}^{2}}
$$

where $P_{r}=\frac{v}{\kappa}$ is the Prandtl number with $v$ the kinematic viscosity $\frac{\mu}{\rho}$ and $\kappa$ the thermometric conductivity $\frac{k}{\rho C_{p}}, F_{r}=\frac{U_{0}^{2}}{a g}$ is the Froude number based on the jet velocity on its surface and $\Delta T=T_{w}-T_{0}$. Equation (12) implies that the pressure across the film remains constant. In the absence of external pressure gradients and with zero shear assumed on the free surface, the pressure term in (11) is identically zero.

The boundary conditions now read

$$
\begin{aligned}
& \bar{U}=\bar{V}=0 \quad \text { on } \bar{Y}=0,0 \leq x \leq \pi \\
& \bar{\phi}=0 \quad \text { on } \bar{Y}=0,0 \leq x \leq \pi \\
& \frac{\partial \bar{U}}{\partial \bar{Y}}=0 \quad \text { at } \bar{Y}=\bar{H}(x), 0 \leq x \leq \pi \\
& \frac{\partial \bar{\phi}}{\partial \bar{Y}}=0 \quad \text { at } \bar{Y}=\bar{H}(x), 0 \leq x \leq \pi \\
& \int_{0}^{\bar{H}(x)} \bar{U} d \bar{Y}=\frac{R_{e}^{\frac{1}{2}} H_{0}^{2}}{2 a^{2} \sin x} \text { for } 0 \leq x \leq \pi .
\end{aligned}
$$

Approximations of equations (10)-(11) under boundary conditions (14), (16) and (18) have been outlined by Gribben [2].

\section{NUMERICAL SOLUTIONS}

The continuity equation (10) can be eliminated by introducing a stream function $\psi$ defined by

$$
\bar{U}=\frac{1}{\sin x} \frac{\partial \psi}{\partial \bar{Y}}, \bar{V}=-\frac{1}{\sin x} \frac{\partial \psi}{\partial x} .
$$

Owing to the geometry, $\bar{H}(x)$ is singular at $x=0$ and $x=\pi$. To remove this singularity, $y$ and $\bar{h}(x)$ are introduced and given by

$$
y=\sin x \bar{Y}, \bar{h}(x)=\sin x \bar{H}(x) .
$$

Substituting equations (19) and (20) into (10)-(18) gives

subject to boundary conditions

$$
\begin{gathered}
\frac{\partial^{3} \psi}{\partial y^{3}}+\frac{1}{F_{r} \sin x}=\left(\frac{1}{\sin ^{2} x}\right)\left(\frac{\partial \psi}{\partial y} \frac{\partial^{2} \psi}{\partial x \partial y}-\frac{\partial \psi}{\partial x} \frac{\partial^{2} \psi}{\partial y^{2}}\right) \\
\frac{\partial^{2} \bar{\phi}}{\partial y^{2}}=\left(\frac{P_{r}}{\sin ^{2} x}\right)\left(\frac{\partial \psi}{\partial y} \frac{\partial \bar{\phi}}{\partial x}-\frac{\partial \psi}{\partial x} \frac{\partial \bar{\phi}}{\partial y}\right)
\end{gathered}
$$




$$
\begin{gathered}
\psi=0, \frac{\partial \psi}{\partial y}=0, \bar{\phi}=0 \quad \text { on } y=0,0 \leq x \leq \pi \\
\psi=\frac{R_{e}^{\frac{1}{2}} H_{0}^{2}}{2 a^{2}}, \frac{\partial^{2} \psi}{\partial y^{2}}=0, \frac{\partial \bar{\phi}}{\partial y}=0 \quad \text { on } y=\bar{h}(x), 0 \leq x \leq \pi \\
\bar{h}=\frac{R_{e}^{\frac{1}{2}} H_{0}^{2}}{2 a^{2}}, \psi=y, \bar{\phi}=1 \quad \text { on } x=0,0<y \leq \frac{R_{e}^{\frac{1}{2}} H_{0}^{2}}{2 a^{2}}
\end{gathered}
$$

where the initial condition (25) appears due to the original initial condition

$$
H=-a+\sqrt{a^{2}+\frac{H_{0}^{2}}{\sin \theta}}, v_{\theta}=U_{0}, T=T_{0} \quad \text { on } \theta=0, a<r \leq \sqrt{a^{2}+\frac{H_{0}^{2}}{\sin \theta}} .
$$

The detailed numerical method procedure for this case is fully discussed in [4,5]. For the sphere, the relevant physical parameters should be chosen as

$$
F(x)=\frac{1}{F_{r} \sin x}, G(x)=\frac{1}{\sin x}, x_{s}=\pi, \gamma=\frac{R_{e}^{\frac{1}{2}} H_{0}^{2}}{2 a^{2}}, \alpha=\frac{2}{3}, \beta=0 .
$$

\section{RESULTS}

A typical run has a coarse grid of dimensions $60 \times 48$ in the $(\xi, \eta)$ domain with each cell being divided into $1,2,3$ and 4 sub-cells respectively. Because of the coordinate singularity at $\xi=0, \eta=1$, a non-uniform grid is employed and given by $\xi=\bar{\xi}^{1.75}, \quad \eta=1-(1-\bar{\eta})^{1.5}$ where $\bar{\xi}$ and $\bar{\eta}$ are uniform. When $\Delta \bar{\xi} \equiv \frac{1}{59} \pi^{\frac{6}{7}}$ and $\Delta \bar{\eta} \equiv \frac{1}{47}$, this gives $\Delta \xi \sim 0.004$ and $\Delta \eta \sim 0.003$ near the singularity, which is sufficiently small to give good accuracy. From the convergence of the extrapolation process, the absolute error is $6 \times 10^{-7}$. A typical set of numerical data is presented in Table.

In Fig. 2, the numerical solution for the film thickness distribution over the sphere is compared with Gribben's approximation [2] for $F_{r}=1$ and $\gamma=0.5$. The agreement is seen to be surprisingly good. Figs. 3-9 depict the flavour of the numerical results. Figs. 3-4, Figs. 5-6 and Figs. 7-9 show film thickness, free surface velocity and free surface temperature respectively for various cases.

For the sphere case, the velocity of the flow is controlled by two opposing forces, viscosity trying to slow it down and gravity trying to speed it up. The viscous component of force affecting the flow is greatest near $x=\frac{\pi}{2}$ and least near $x=0$ and $x=\pi$. Figs. 5 and 6 show that free surface velocity initially has a slight increase, followed by a sharp decrease as viscosity starts to dominate, and finally a gradual increase is observed as the bottom of the sphere is approached. As $F_{r}$ decreases, 
the effect of gravity increases and hence the thin film thickness, high velocity and low temperature appear corresponding to the small $F_{r}$ values. As $\gamma$ decreases, the amount of fluid in the impinging jet decreases and the ensuing film becomes thinner. The effect of viscosity increases and hence the low velocity and low temperature appear corresponding to the small $\gamma$ values. Where $\gamma$ is greater than a certain value, e.g., $\gamma=2$ for $F_{r}=1$, Fig. 6 shows no decrease for the velocity over the sphere. As $P_{r}$ increases, the temperature decrease becomes more gradual.

\section{CONCLUDING REMARKS}

Comprehensive numerical solutions for the flow of cold axi-symmetric jets over a hot sphere have been obtained. The gross features of such flows have been illustrated over a range of representative parameter values. In a practical setting, appropriate parameter values may be evaluated and the design characteristics readily identified from the numerical scheme. In practice, it is not obvious that uniform wetting of the sphere would occur. Instabilities may distort or even disrupt such a uniform distribution. Nevertheless for a given overall flow rate, the model may represent a valuable first approximation to the aggregate properties of the flow [6-13].

\section{REFERENCES}

[1] Gyure, D.C. and Krantz, W.B., "Laminar film flow over a sphere", Industrial \& Engineering Chemistry Fundamentals, Vol. 22, No. 4, pp. 405-410, (1983).

[2] Gribben, R.J., "Laminar film flow over a sphere at high Reynolds number", Mathematical Engineering Industry, Vol. 1, No. 4, pp. 279-288, (1987).

[3] Hunt, R., "The numerical solution of parabolic free boundary problems arising from thin film flows", Journal of Computational Physics, Vol. 84, No. 2, pp. 377402, (1989).

[4] Shu, J.-J. and Wilks, G., "Heat transfer in the flow of a cold, two-dimensional draining sheet over a hot, horizontal cylinder", to appear.

[5] Shu, J.-J. and Wilks, G., "An accurate numerical method for systems of differentio-integral equations associated with multiphase flow", Computers \& Fluids, Vol. 24, No. 6, pp. 625-652, (1995).

[6] Shu, J.-J. and Wilks, G., "Heat transfer in the flow of a cold, two-dimensional vertical liquid jet against a hot, horizontal plate", International Journal of Heat and Mass Transfer, Vol. 39, No. 16, pp. 3367-3379, (1996).

[7] Shu, J.-J. and Wilks, G., "Mixed-convection laminar film condensation on a semi-infinite vertical plate", Journal of Fluid Mechanics, Vol. 300, pp. 207-229, (1995).

[8] Shu, J.-J. and Pop, I., "Inclined wall plumes in porous media", Fluid Dynamics Research, Vol. 21, No. 4, pp. 303-317, (1997).

[9] Shu, J.-J. and Pop, I., "Transient conjugate free convection from a vertical flat plate in a porous medium subjected to a sudden change in surface heat flux", International Journal of Engineering Science, Vol. 36, No. 2, pp. 207-214, (1998). 
[10] Shu, J.-J. and Pop, I., "On thermal boundary layers on a flat plate subjected to a variable heat flux", International Journal of Heat and Fluid Flow, Vol. 19, No. 1, pp. 79-84, (1998).

[11] Shu, J.-J., and Pop, I., "Thermal interaction between free convection and forced convection along a conducting plate embedded in a porous medium", Hybrid Methods in Engineering: --- Modeling, Programming, Analysis, Animation, Vol. 1, No. 1, pp. 55-66, (1999).

[12] Shu, J.-J. and Pop, I., "Thermal interaction between free convection and forced convection along a vertical conducting wall", Heat and Mass Transfer, Vol. 35, No. 1, pp. 33-38, (1999).

[13] Shu, J.-J., "Microscale heat transfer in a free jet against a plane surface", Superlattices and Microstructures, Vol. 35, No. 3-6, pp. 645-656, (2004). 


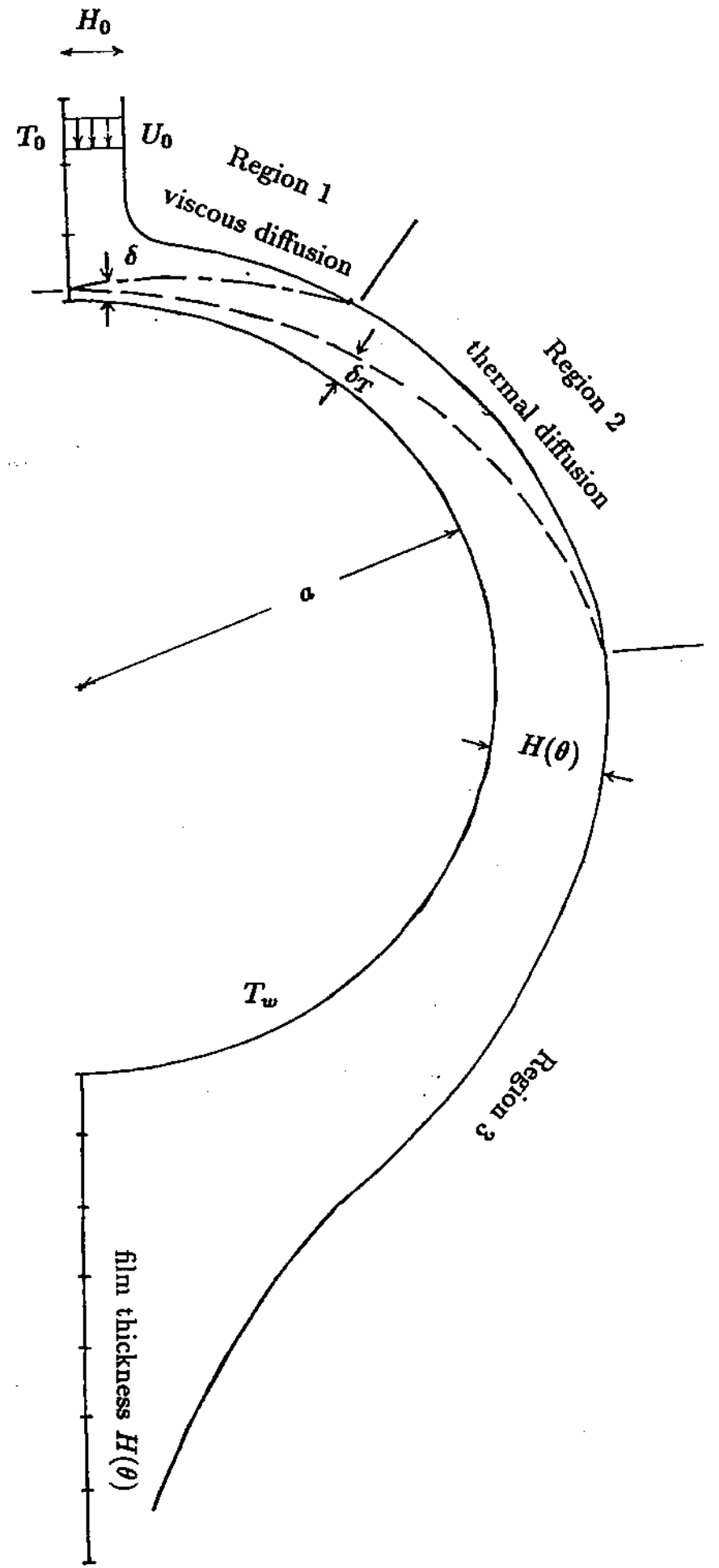

Fig. 1. The vertical jet and resultant film for the sphere 


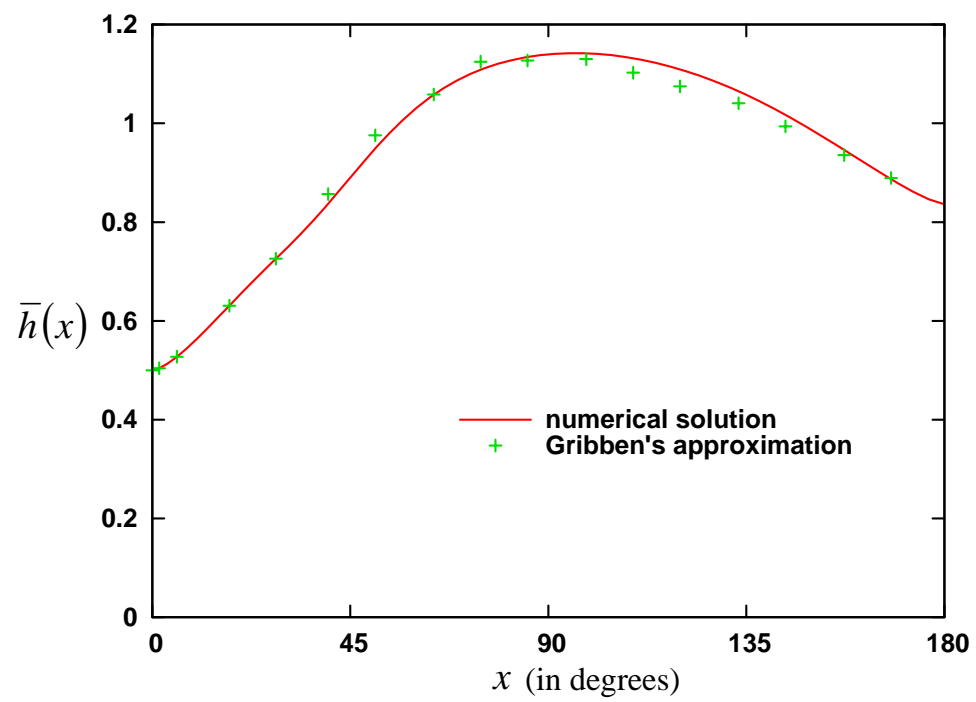

Fig. 2. Film thickness for the numerical solution and Gribben's approximation at $F_{r}=1$ and $\gamma=0.5$

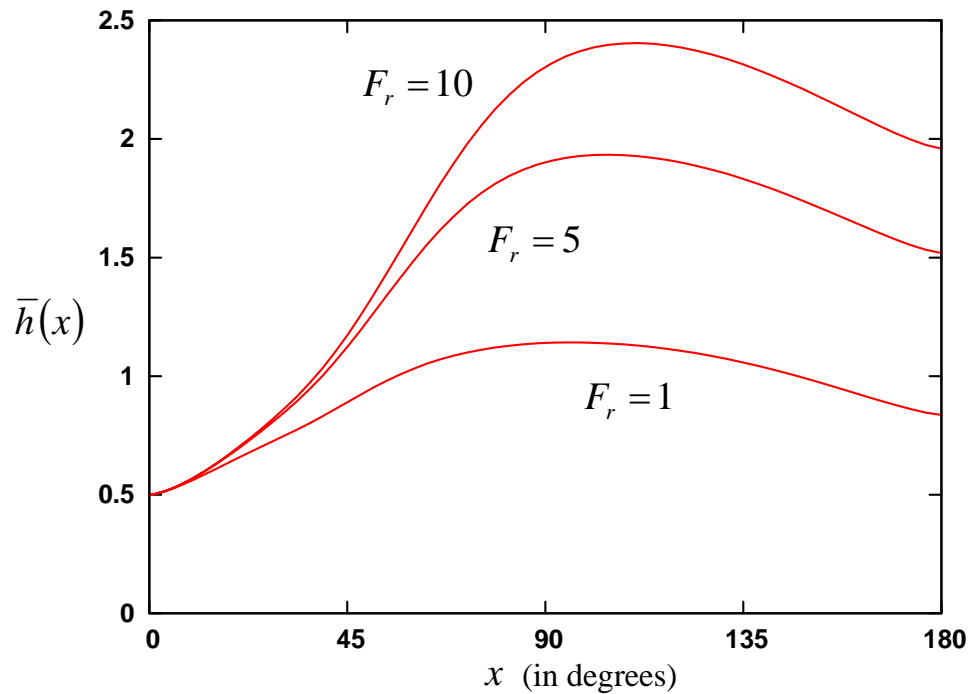

Fig. 3. Film thickness for various Froude numbers at $\gamma=0.5$ 


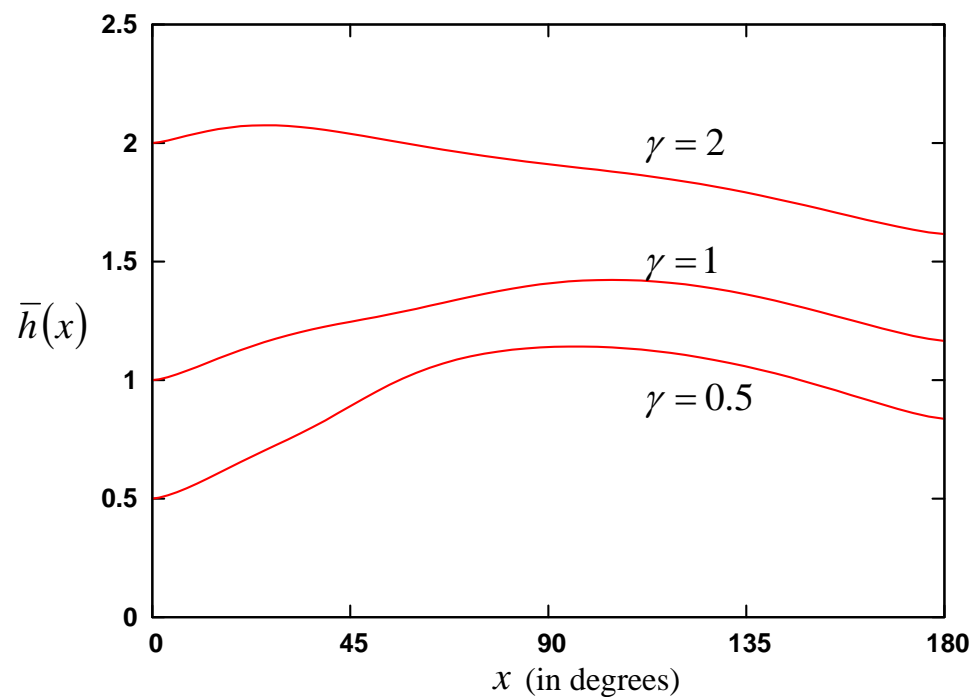

Fig. 4. Film thickness for various values of the parameter $\gamma$ at $F_{r}=1$

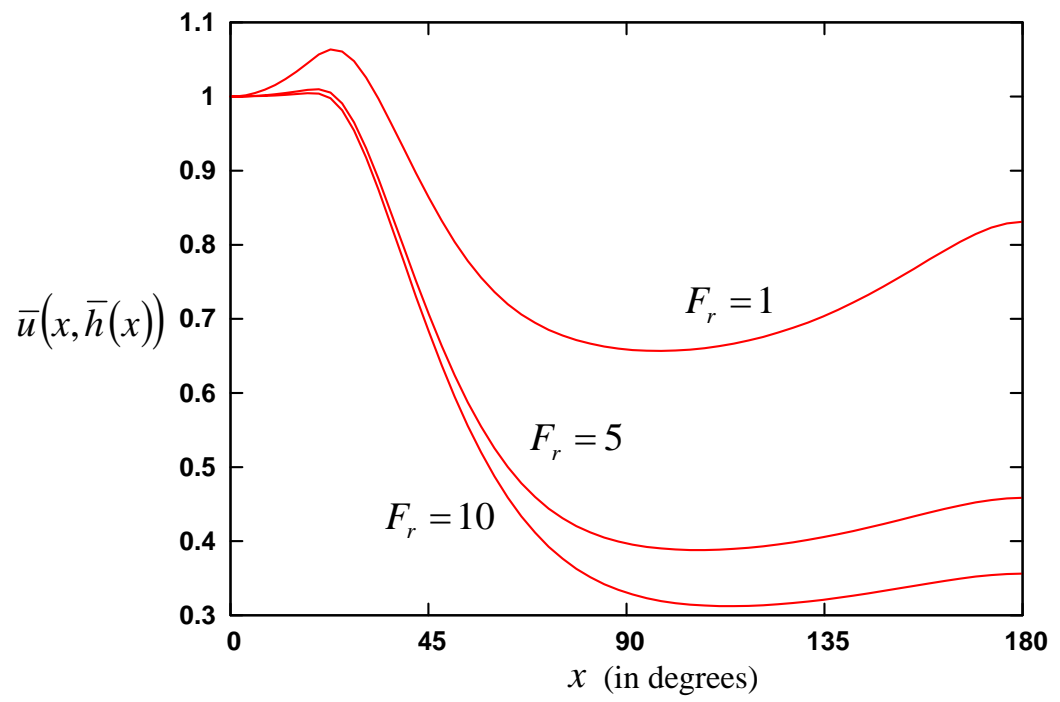

Fig. 5. Free surface velocity for various Froude numbers at $\gamma=0.5$ 


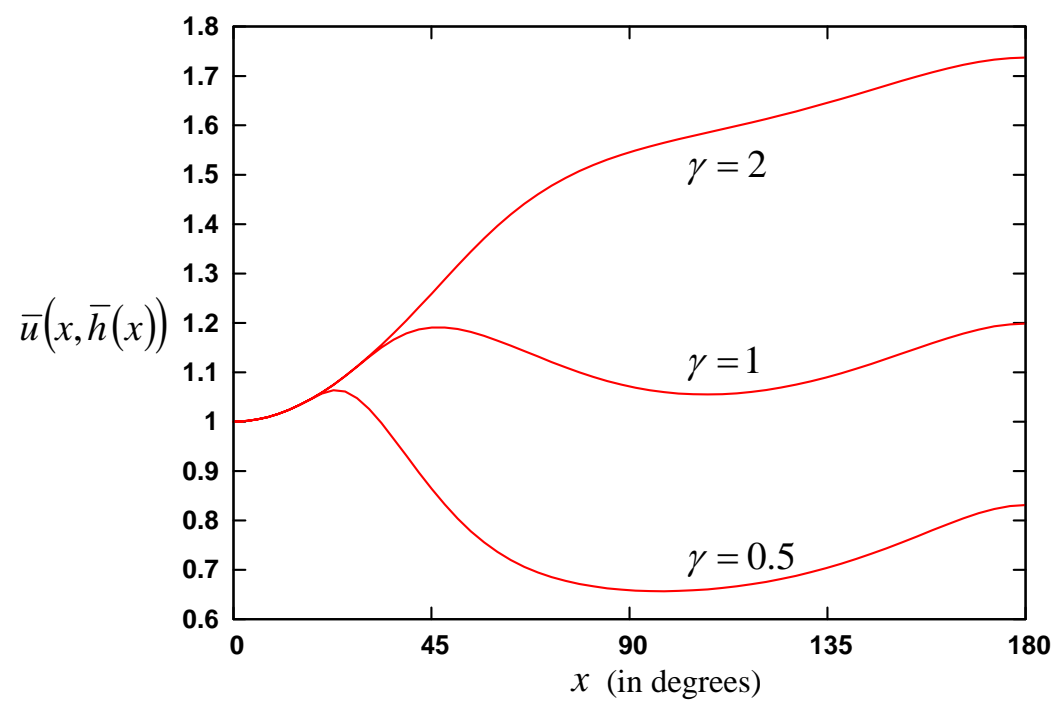

Fig. 6. Free surface velocity for various values of the parameter $\gamma$ at $F_{r}=1$

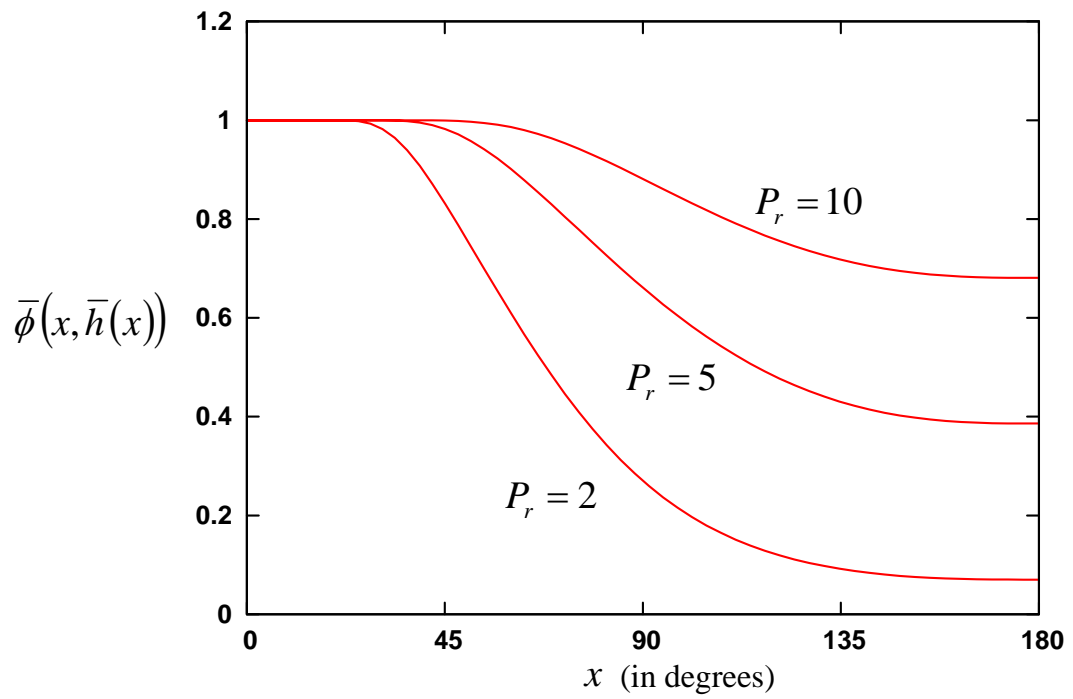

Fig. 7. Free surface temperature for various Prandtl numbers at $F_{r}=1$ and $\gamma=0.5$ 


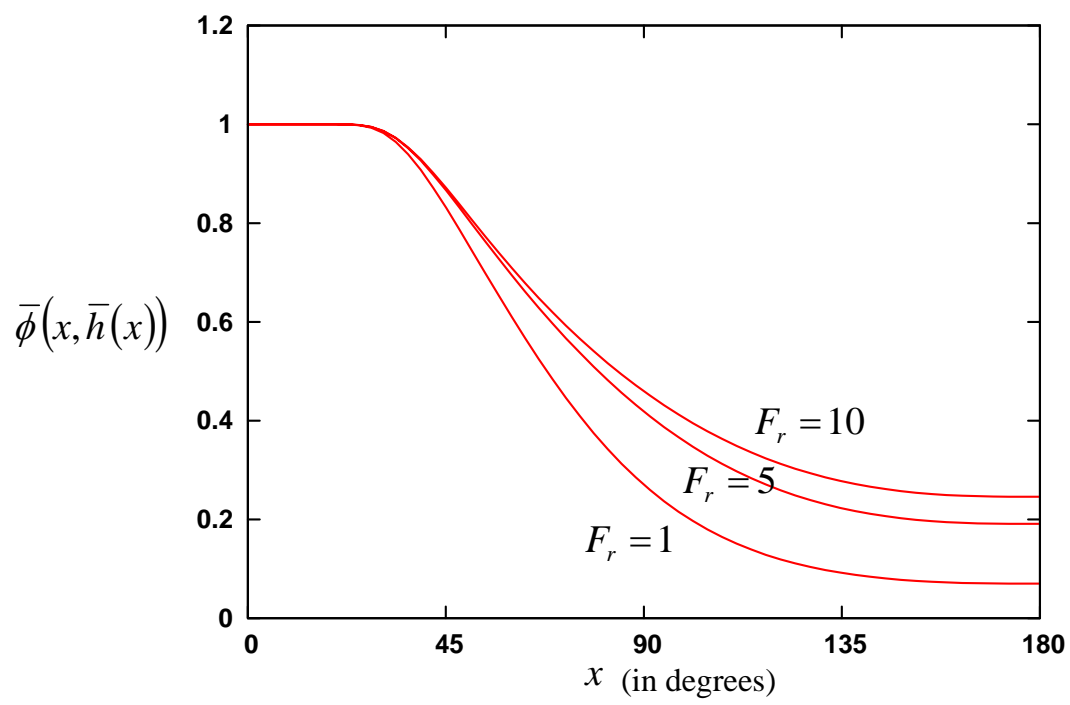

Fig. 8. Free surface temperature for various Froude numbers at $\gamma=0.5$ and $P_{r}=2$

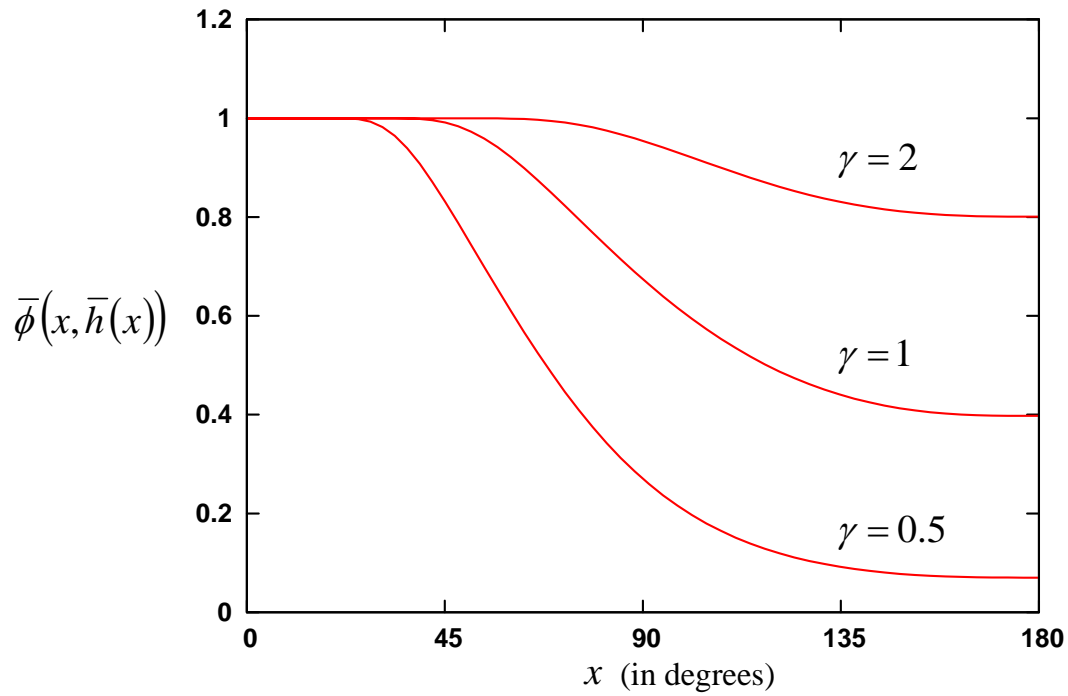

Fig. 9. Free surface temperature for various values of the parameter $\gamma$ at $F_{r}=1$ and

$$
P_{r}=2
$$


Table. Film thickness, free surface velocity and temperature for the sphere with

$$
F_{r}=1, \gamma=0.5 \text { and } P_{r}=2
$$

\begin{tabular}{||c|c|c|c||}
\hline$x$ & $\begin{array}{c}\text { film } \\
\text { thickness } \\
\bar{h}(x)\end{array}$ & $\begin{array}{c}\text { free surface } \\
\text { velocity } \\
\bar{u}(x, \bar{h}(x))\end{array}$ & $\begin{array}{c}\text { free surface } \\
\text { temperature } \\
\bar{\phi}(x, \bar{h}(x))\end{array}$ \\
\hline 0.000 & 0.500 & 1.000 & 1.000 \\
0.218 & 0.585 & 1.023 & 1.000 \\
0.396 & 0.678 & 1.063 & 1.000 \\
0.587 & 0.775 & 0.997 & 0.964 \\
0.786 & 0.891 & 0.864 & 0.831 \\
0.994 & 1.007 & 0.756 & 0.654 \\
1.208 & 1.086 & 0.695 & 0.484 \\
1.427 & 1.128 & 0.667 & 0.343 \\
1.595 & 1.141 & 0.658 & 0.260 \\
1.823 & 1.138 & 0.659 & 0.180 \\
1.996 & 1.122 & 0.667 & 0.139 \\
2.290 & 1.072 & 0.695 & $9.760 \times 10^{-2}$ \\
2.590 & 0.994 & 0.744 & $7.779 \times 10^{-2}$ \\
3.018 & 0.861 & 0.823 & $7.022 \times 10^{-2}$ \\
$\pi$ & 0.836 & 0.831 & $7.013 \times 10^{-2}$ \\
\hline
\end{tabular}

